\title{
Configuring use-oriented aero-engine overhaul service with multi-objective optimization for environmental sustainability
}

Huibin Sun, Yang Liu, Tomohiko Sakao and Zhan Wang

The self-archived postprint version of this journal article is available at Linköping University Institutional Repository (DiVA):

http://urn.kb.se/resolve?urn=urn:nbn:se:liu:diva-133159

N.B.: When citing this work, cite the original publication.

Sun, H., Liu, Y., Sakao, T., Wang, Z., (2017), Configuring use-oriented aero-engine overhaul service with multi-objective optimization for environmental sustainability, Journal of Cleaner Production, 162, S94-S106. https://doi.org/10.1016/j.jclepro.2016.12.022

Original publication available at:

https://doi.org/10.1016/j.jclepro.2016.12.022

Copyright: Elsevier

http://www.elsevier.com/

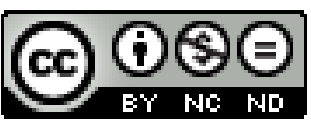




\section{Accepted Manuscript}

Configuring use-oriented aero-engine overhaul service with multi-objective optimization for environmental sustainability

Huibin Sun, Yang Liu, Tomohiko Sakao, Zhan Wang

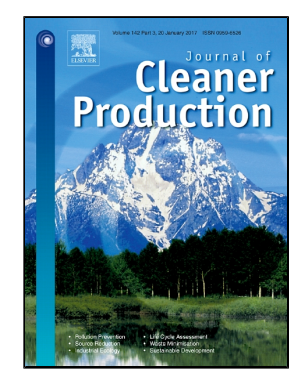

PII:

S0959-6526(16)32077-7

DOI:

10.1016/j.jclepro.2016.12.022

Reference:

JCLP 8597

To appear in:

Journal of Cleaner Production

Received Date:

28 July 2015

Revised Date:

01 December 2016

Accepted Date:

06 December 2016

Please cite this article as: Huibin Sun, Yang Liu, Tomohiko Sakao, Zhan Wang, Configuring useoriented aero-engine overhaul service with multi-objective optimization for environmental sustainability, Journal of Cleaner Production (2016), doi: 10.1016/j.jclepro.2016.12.022

This is a PDF file of an unedited manuscript that has been accepted for publication. As a service to our customers we are providing this early version of the manuscript. The manuscript will undergo copyediting, typesetting, and review of the resulting proof before it is published in its final form. Please note that during the production process errors may be discovered which could affect the content, and all legal disclaimers that apply to the journal pertain. 
Configuring use-oriented aero-engine overhaul service with multi-objective optimization for environmental sustainability

\author{
Huibin Suna, ${ }^{a}$, Yang Liu ${ }^{b, c,{ }^{*}}$, Tomohiko Sakao ${ }^{b}$, Zhan Wang ${ }^{a}$ \\ a Laboratory of Contemporary Design and Integrated Manufacturing Technology, Ministry of \\ Education, Northwestern Polytechnical University, Xi'an, P. R. China \\ ${ }^{b}$ Division of Environmental Technology and Management, Department of Management and \\ Engineering, Linköping University, SE-581 83 Linköping, Sweden \\ c Department of Production, University of Vaasa, PL 700, 65101 Vaasa, Finland \\ Corresponding Authors: sun_huibin@nwpu.edu.cn (H. Sun), yang.liu@liu.se (Y. Liu)
}

Abstract: As a typical sustainable product-service system, the aero-engine overhaul service aims to recover the functionality to fulfil certain flight hours and thrust requirements. How to configure the overhaul service with various sub-services is a key issue in industry. In this paper, a use-oriented aero-engine overhaul service configuration approach is proposed with two methods: overhaul service configuration and part repair service configuration. The overhaul service configuration method decomposes the overhaul service into various subservices and creates a hierarchical service tree model, taking the cost and energy consumption into consideration. Based on the sub-service filtering and selection rule, the bill of exchange service comes with the bill of discard service. The part repair service configuration method matches each part repair procedure service with a proper service provider. Some non-dominated bills of repair services are obtained by minimizing the repair service cost, finishing time deviation and energy consumption. Considering the multiobjective character of the overhaul service configuration problem, these two methods result in some Pareto solutions to fulfil the service requirements. Through implementing the approach as a prototype, a case study is shown to illustrate the use-oriented aero-engine overhaul service configuration approach and verify its feasibility. A bridge between the flight hours and thrust requirements and the overhaul service configuration results is built when the cost and energy consumption are minimized. The study contributes to the shift of aeroengine overhaul services from the add-on services to sustainable use-oriented productservice systems.

Keywords: use-oriented; product-service systems; configuration; optimization; sustainability

\title{
1. Introduction
}

At present, environmental issues are widely recognized as important throughout the world. Enterprises try to create more value by making most use of limited natural resources (Tukker, 2015). In addition, more and more enterprises are trying to increase their business areas over value chains and provide combination of products and services instead of providing physical products only (Mont et al., 2006; Meir et al., 2010). Under this circumstance, sustainable product-service systems (PSSs) attract industry as new ways of doing more sustainable business (Mont, 2002; Sakao et al., 2007). A sustainable PSS is defined as "a system of products, services, supporting networks and infrastructure designed to be competitive, satisfy customer needs and have lower environmental impact than traditional business models" (Mont, 2002). The sustainable PSSs are believed to re-orient the current standards of consumption and production, and to enable a move towards a 
more sustainable society (UNEP, 2002; Tukker, 2015). Offering sustainable PSSs has positive potential for economic, social, and environmental effects as companies could improve resource utilization, competitiveness, sustainability, and eco-efficiency at the same time (Liu, 2013; Liu and Liang, 2015; Chou et al., 2015).

Different categories of PSSs exist in industry. According to Tukker (2004), PSSs are classified into product-oriented, use-oriented, and result-oriented ones. Product-oriented PSSs include supplying consumables to the provided products. Use-oriented PSSs include product leasing with the provider's responsibility to maintain the product functions, as well as product sharing/pooling (Mont and Tukker, 2006). Sharing/pooling utilizes less resources for a given need and thus benefits the environment (Byers et al., 2015), but is not a subcategory targeted in this paper. Comparing product-oriented PSSs and product leasing, which is the targeted sub-category of use-oriented PSSs in this paper, the latter improves the environmental performance more (Tukker, 2004). A main reason for this is that a provider of use-oriented PSSs often has responsibility to maintain the functionality and incentive to increase efficiency especially in the use phase.

Regarding the aero-engine sector, product- and use-oriented PSSs are provided (Smith 2013). Under the use-oriented PSSs, the consumers purchase the aero-engines' flight hour services. The aero-engines owned by the service provider, are only service carriers. Among different service activities, overhaul is a significant issue in the maintenance, repair, and overhaul (MRO) chain (Zhu et al., 2012). The use-oriented aero-engine overhaul service aims to recover or rebuild the functionality to fulfil certain flight hour and thrust requirement in the following service cycle.

Although potential benefits of a use-oriented aero-engine overhaul service have been highlighted above, insights from literatures about PSS are often described in a generic level (Tukker, 2015) and thus insights for concrete ways to practice this service efficiently is limited (Vezzoli et al, 2015). In more details, the following barriers are faced.

- An aero-engine overhaul service is a complex activity (Zhu et al., 2012) and may benefit from introducing a hierarchical structure (Joore and Brezet, 2015). However, a concrete way to configure the overhaul service with variable subservice options in an optimal manner on the context of sustainable PSS is not established.

- To practise an aero-engine overhaul service efficiently, selecting the best or better service and thus evaluating alternative services with multiple indexes is needed. Besides the flight hours and thrust requirements, cost and environmental impacts should be considered. However, a concrete evaluation method for this purpose is missing.

Therefore, to solve these problems, this paper aims to propose a use-oriented aeroengine overhaul service configuration approach. More specifically, a bridge between the requirements for the service and the configurations is built. The configuration process takes environmental impacts into account and adopts multi-objective evaluation and optimization algorithm. Two key issues, overhaul service configuration and part repair service configuration, are presented in detail.

The rest of this paper is organized as follows. Section 2 reviews literature related to service configuration and evaluation. Section 3 puts forward the use-oriented aero-engine 
overhaul service configuration and evaluation approach. In Sections 4 and 5, the overhaul service configuration method and the repair service configuration method are presented respectively. In Section 6, how to implement the approach as a prototype is presented. The case study is shown in Section 7, which is followed by concluding remarks in Section 8.

\section{Literature review}

With background of diversification of customer demands (Koren 2010), efficient customization receives attention (Hu et al., 2011) - even in the environmental sustainability field (Sakao and Fargnoli 2010). Combined with servitisation trend in industry (Needly, 2007; Meier et al., 2010), PSS customization is a relevant issue in industry (Zhang et al., 2016a). To customize PSSs, configuration of product and service modules in PSSs is an efficient approach (Song and Sakao, 2017).

To configure PSSs, several approaches have been proposed. Shen et al. (2012) proposed an ontology-based approach and a system for representing knowledge of configuring product extension services in servitisation. Long et al. (2013) proposed an approach in which functional needs and perception needs of customers were translated into specific PSS configurations through factor analysis and a sort of statistical learning theory. Some other approaches were based on a hierarchical systems optimization method (Cherubini et al., 2015). Among them, the embedded analytical target cascading (ATC) mechanism (Kim et al., 2000) is used optimal system design (Kim, 2001) and supply chain configuration (Qu et al, 2009). In addition, an extended ATC method was used to accommodate "OR" elements, in terms of working logic and mathematical formulation (Huang et al., 2008). The alternative selection of "OR" elements could be integrated into the target cascading process. An embedded ATC mechanism could coordinate all the related services to formulate the optimal solution (Qu et al., 2009; Huang et al., 2011). This hierarchical service configuration method was verified to be more efficient than a centralized one. Further, a PSS based on warehouse service was described as a tree structure by using ATC and an optimal resource configuration was obtained (Cao and Jiang, 2013).

When a service is configured, some quantifiable objectives regarding its performance may be determined (Aurich et al., 2009). This can be formalized as a service composition and optimal-selection problem (Tao et al., 2011). Different objectives should be selected according to the case in question, for example, cost and $\mathrm{CO}_{2}$ emission. Some researchers argued that product and/or service degradation must simultaneously consider users' expectations and environmental aspects (Gaiardelli et al., 2014; Salazar et al., 2015). However, most of earlier researches adopted single-objective combinational optimization problem formulation. Some algorithms were designed to solve multi objective problems. Typical examples included the group leader algorithm with the idea of Pareto solution (Xiang et al., 2014), the quantum multi-agent evolutionary algorithm (Tao et al., 2014), the uncertainty and genetic algorithm-based (Huang et al., 2011), the chaos optimal algorithm (Huang et al., 2014), etc.

A product service could be hierarchically decomposed into some sub-services based on ATC method. Some objectives were considered to evaluate every solution. Most works aimed to decompose the top service into sub-services for target cascading or task disassembling ( $Q u$ et al., 2010; Huang et al., 2008). In fact, a use-oriented aero-engine 
overhaul service is expected to fulfil certain service requirements. The configuration process should decompose the overhaul service into various sub-services when both the service requirements and other factors are considered. A bridge between service requirements and service configurations is needed. Considering the trend of the environmental awareness, the ecological impacts should be considered as well.

\section{The use-oriented aero-engine overhaul service configuration approach}

\subsection{Problem and assumptions}

The overhaul service configuration aims to find out some optimized combinational service solutions by decomposing the use-oriented overhaul service into various sub-services. Assuming $X$ is a solution, the problem can be formulated as follow.

$$
\text { Minimize } C(X), E(X)
$$

$$
\text { Subject to } X \in R, L(X) \geq L, P(X) \geq P
$$

Here, $C(X)$ and $E(X)$ are the overhaul service cost and energy consumption respectively. $L(X)$ and $P(X)$ are actual flight hour and thrust respectively. $R$ is the set of all possible solutions. $L$ and $P$ stand for the flight hours and thrust requirements respectively.

\subsection{Problem solving procedure}

To solve the problem, a use-oriented aero-engine overhaul service configuration approach is proposed as Fig. 1 shows. The following two steps are regarded as its key issues.

1) Overhaul service configuration. A use-oriented aero-engine overhaul service can be decomposed into and configured by various sub-services. Each sub-service may have one or more options, including repair, exchange, and discard (RED) as follows.

- The repair option means recovering a part's or a component's mechanical functionality and making it work again.

- The exchange option means withdrawing a part or a component and replacing it with another new one or used one. The replaced part or component may be reused one day.

- The discard option means recycling a part's or a component's material and replacing it with a new one or used one.

Considering the service requirements, each part's or component's qualified options can be filtered. A configuration rule is needed to select the best option from the remaining options for each part or component. The total overhaul cost and energy consumption are going to be minimized when the flight hours and thrust constraints apply. The bill of exchange services come with the bill of discard services.

2) Part repair service configuration. Accordingly, some part repair services are going to be decided. Each of them is composed of several repair procedures. Outsourcing these procedures to proper service providers is a vital problem. Here, the total repair cost, finishing time deviation and energy consumption are going to be minimized, when the sequence constraint and resource constraint apply. A multi-objective optimization model 
and the corresponding algorithm are put forward. Some bills of repair services can be resolved.

Finally, some bills of services are obtained because of the problem's multi-objective character. The use-oriented overhaul service requirement can be fulfilled by these service configuration results. Two methods mentioned above are going to be discussed in detail in the following sections.

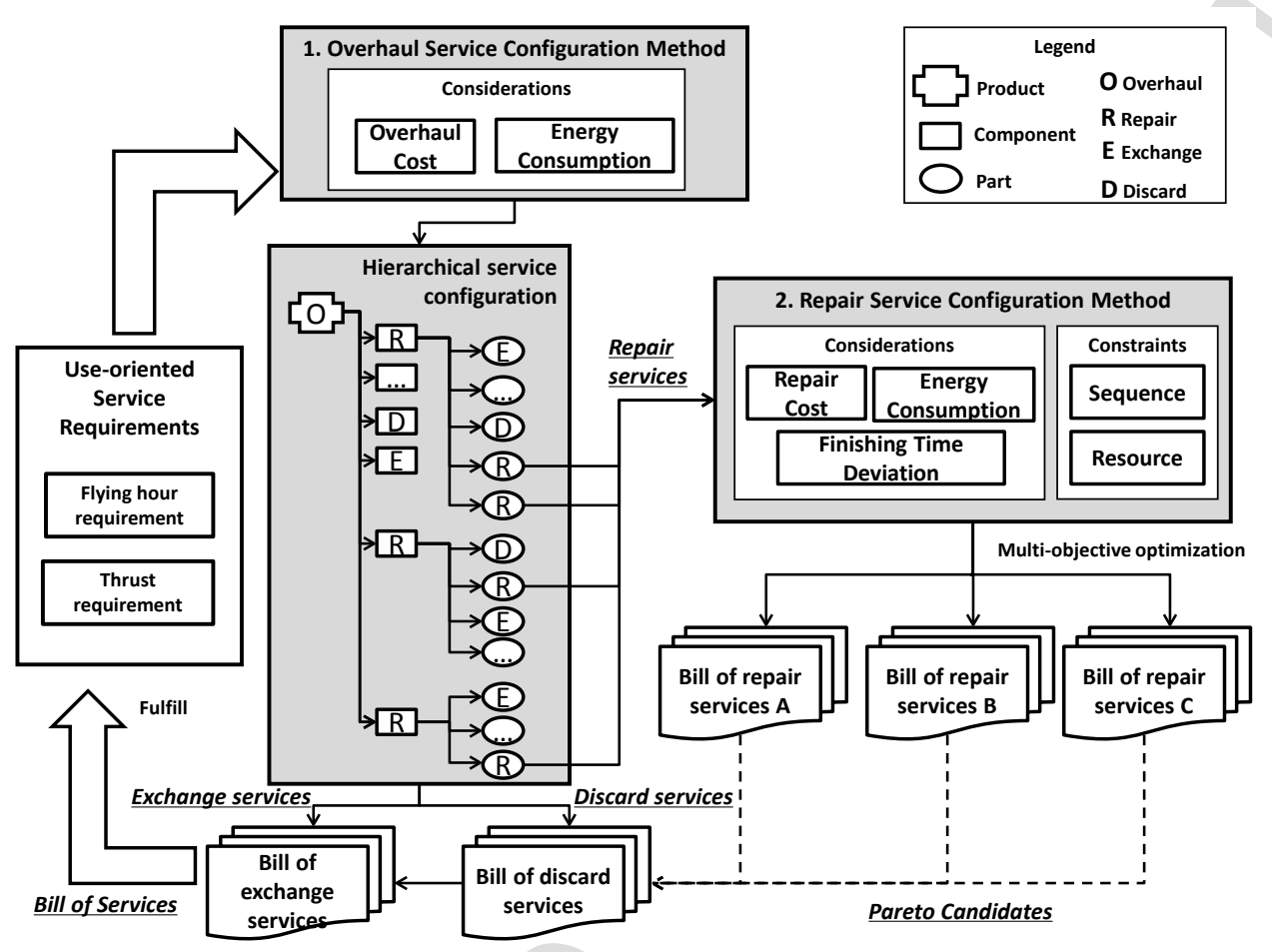

Fig. 1 The use-oriented aero-engine overhaul service configuration approach

\section{Overhaul service configuration method}

\subsection{Hierarchical service modelling}

This method aims to convert the overhaul service problem into a hierarchical service tree model (HSTM). The top overhaul service is decomposed into a set of sub-services. This recursive composition repeats until the bottom-level sub-services do not have further subservices. To decompose the top overhaul service, the AND connection and OR connection are defined.

1) The AND connection describes the must-be included sub-service relationship. A parent service node is enabled only when all its sub-service nodes are satisfied. For example, prior to finishing an assembly service, all services related to the necessary parts must be done.

2) The OR connection describes the one-of service relationship. A parent service node is enabled when one of its sub-service nodes is satisfied. If a service has some options, such as repair, exchange and discard, an OR connection can be used. A decision about the options should be made. 
Based on the AND and OR connections, a use-oriented overhaul service can be decomposed from top to down into the following three layers.

- Aero-engine overhaul service layer. There is an aero-engine overhaul service node only in this layer. Its goal is in line with the use-oriented aero-engine overhaul service.

- Component repair service layer. Sub-service nodes of the aero-engine overhaul service node are included. Components' service options, including repair service, exchange service and discard service are going to be decided.

- Part repair service layer. Sub-service nodes of the overhaul and component overhaul service nodes are included. Parts' service options, such as repair, exchange, and discard, are going to be decided.

According to these rules, a typical topology of the hierarchical service tree model (HSTM) can be obtained as Fig. 2 shows. Rectangles are service nodes. Each arrow stands for a connection. The arrows for an OR connection are adjoined by the same numbered circles. Other arrows stand for AND connections.

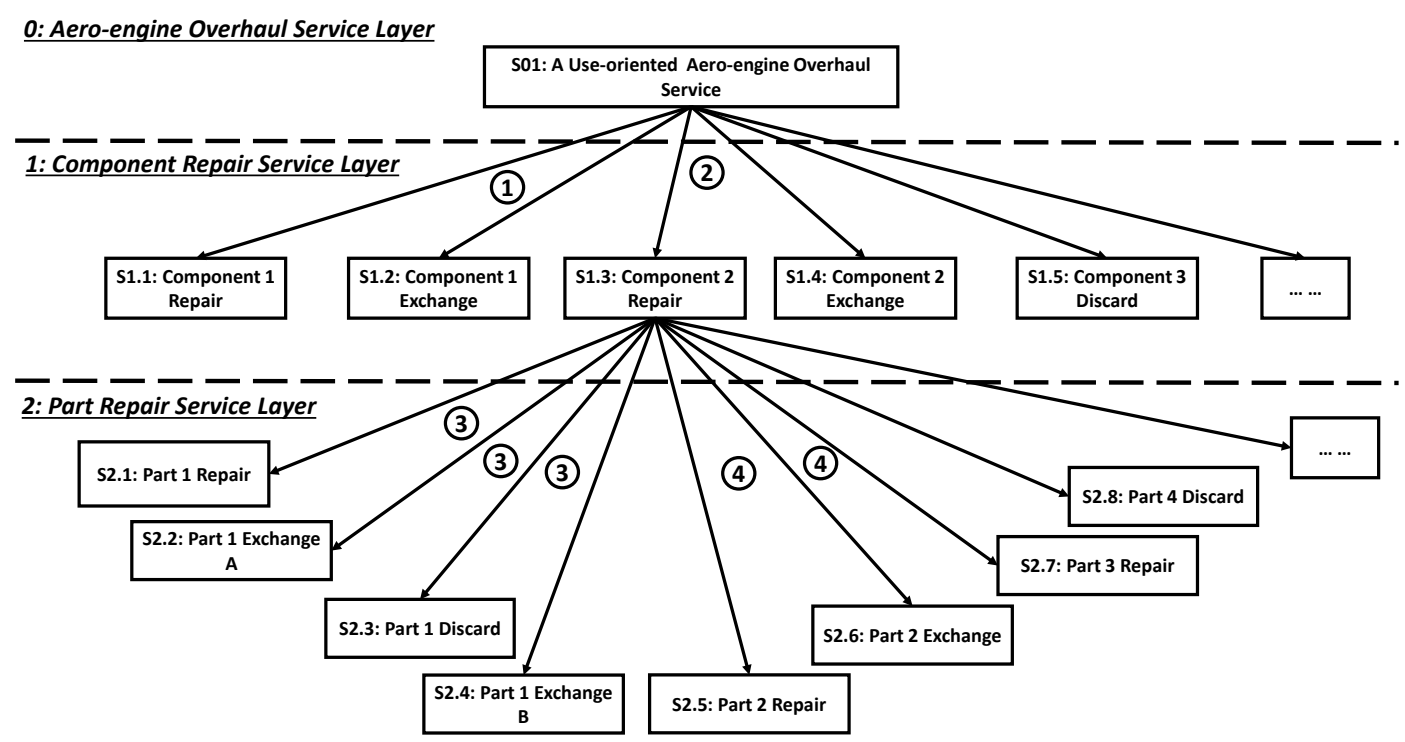

Fig. 2 A hierarchical service tree example

An HSTM with $N$ nodes can also be described clearly by matrices. Some definitions are given as follows.

Definition 1: the original topology of the HSTM can be described by a service relationship matrix $Z=\left[z_{i j}\right]_{N \times N}$. Each row or column stands for a service node in sequence. The value of the element $z_{i j}$ can be decided according to the following rules.

- 1 : service node $j$ is a child of service node $i$ definitely when an AND connection exists.

- $\quad 0$ : no direct connection exists between service nodes $i$ and $j$.

- Negative integers: some sub-service nodes with the same negative integer are a group of options for service node $i$ based on OR connections. 
Definition 2: the cost of all service nodes can be summarised by an $\mathrm{N}$-dimension cost vector $Y^{c}$. The element $y_{i}^{c}$ is the cost of service node $i$.

Definition 3: the energy consumption of all service nodes can be summarised by an $\mathrm{N}$ dimension energy consumption vector $Y^{s}$. The element $y_{i}^{s}$ is the energy consumption of service node $i$. It is a middle score in the 1-5 scale (Chou et al., 2015).

Definition 4: the remaining flight hours of all service nodes can be summarised by an $\mathrm{N}$ dimension vector $Y^{l}$. For a service node in the part repair service layer, the value of $y_{i}^{l}$ is determined according to the flight hour history. For a parent node, $y_{i}^{l}$ equals to its children's minimum remaining flight hours.

Definition 5: the actual thrust of all service nodes can be summarised by an $N$ dimension vector $Y^{p}$. For a service node in the part repair service layer, the value of $y_{i}^{p}$ is determined according to the check result. For a parent node, the value equals to its children's minimum thrust.

\subsection{Service node formulation}

When the HSTM is built, the requirements and constraints should also be decomposed from top to down. Prior to this step, a service node can be described by a 5 -tuple as follow.

$$
D_{i}=\left\{C_{i}, c_{i}, e_{i}, l_{i}, p_{i}\right\}
$$

Here, $D_{i}$ is the $i^{\text {th }}$ service node in the hierarchical service model, when all nodes are sorted in top-down and left-right sequence. Normally, the top overhaul service node is the first one. $c_{i}$ is the child node set of node $D_{i} . c_{i}$ and $e_{i}$ are the cost and energy consumption of the service node $D_{i}, l_{i}$ and $p_{i}$ are the actual flight hour and thrust values of service node $D_{i}$ respectively. They are given by the service provider according the part's or component's status and service history. If the expression $l_{i}<L$ or $p_{i}<P$ is true, the service node $D_{i}$ is set as $D_{i}=O$, which means an unqualified node.

\subsection{Decision-making procedure}

The overhaul service configuration is a complex decision-making problem when each service node may have multiple options. To get the configure results efficiently, a decisionmaking procedure is designed and shown in Fig. 3. 


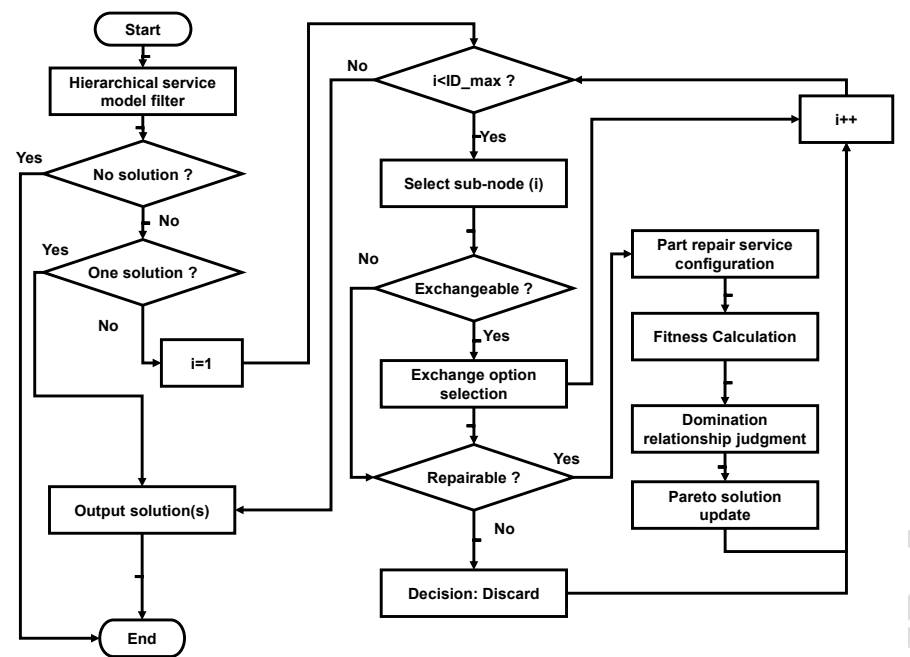

Fig. 3 The overhaul service configuration procedure

Main steps are listed as follows.

1) Hierarchical service model filter. Based on the comparison between the requirements and actual values of flight hours and thrust, all unqualified nodes are filtered. Here, a matrix is defined by $Z^{\prime}=Z$. If the expression $y_{j}^{l}<L$ or $y_{j}^{p}<P$ is true, service node $D_{j}$ and all its sub-service nodes are filtered. Here, $\mathrm{L}$ and $\mathrm{P}$ are respectively flight hours and thrust requirements. Notations $y_{j}^{l}$ and $y_{j}^{p}$ are respectively the remaining flight hours and thrust.

The element $z_{i j}^{\prime}$ is updated according the following rule.

$z_{i j}^{\prime}=0, z^{\prime}{ }_{j k}=0, i \in[1, N), j \in(i, N], k \in(j, N]$

Another matrix is defined by $Z^{\prime \prime}=Z^{\prime}+Z$. If the following expression is true, no solution exists because all options described by OR connections have been filtered.

$$
\forall i \in[1, N], j \in(i, N], k \in(i, N), \exists z^{\prime \prime}{ }_{i j}=e \wedge z^{\prime \prime}{ }_{i k}=e-1, e=-1,-2, \ldots
$$

On the contrary, the variable $v$ is defined as the row number that includes the negative integer no less than 2 times in matrix $Z "$. A small $v$ value means that few combinations exist. No specific algorithm is needed when simple enumeration is good enough to find the optimum solution out of few candidates. Otherwise, the matrix $Z$ ' is regarded as a solution basis. To simplify the calculation, the unique negative integer in each row is set as 1 .

2) Sub-node selection rule

Normally, an exchange option is preferred because it is believed to be most economic and environment friendly. If two or more exchange options under the same OR connection exist, the one that consumes less energy is preferred. If a repair option can be found when no exchange option exists, a complex decision should be made. As to a component, the repair service should be decomposed continuously. As to a part, a service provider should be 
selected for each repair procedure. In fact, it is a combinational problem when all part repair services are optimized at the same time globally. Cost, energy consumption and finishing time deviation are the objectives. Detailed discussion about part repair service configuration is presented in section 5. Finally, discard is the last choice if no exchange or repair options are available. Sometimes, a repair option may consume more money and energy than a discard option, which is not in the scope of this work. The sub-node select step ends when no component repair service node can be decomposed further. In other word, a leaf node in the HSTM should be one of the following five types, including component exchange, component discard, part exchange, part repair and part discard.

3) Fitness calculation. Based on the filtered and configured HSTM, a cost-weighted tree can be built when the element $x_{i j}^{(u)}$ is updated as $x_{i j}^{(u)} y_{j}^{c}$. The overhaul service cost of the solution $X^{(u)}$ can be calculated by the following equation.

$$
c^{(u)}=\sum_{i=1}^{N} \sum_{j=i}^{N} x_{i j}^{(u)} y_{j}^{c}
$$

Similarly, an energy consumption-weighted tree can also be built when the element $x_{i j}^{(u)}$ is updated as $x_{i j}^{(u)} y_{j}^{s}$. The energy consumption of the solution $X^{(u)}$ can be calculated by the following equation.

$$
e^{(u)}=\sum_{i=1}^{N} \sum_{j=i}^{N} X_{i j}^{(u)} y_{j}^{S}
$$

The fitness of solution $X^{(u)}$ is a vector including its service cost and energy consumption as $f^{(u)}=\left[c^{(u)}, e^{(u)}\right]$.

4) Solution selection rule. Due to the multi-objective characteristic of the part repair service configuration, the unique optimum solution may not exist. Multiple bills of repair services could be found. Each of them contributes to a Pareto solution (PS) when the bill of the exchange services and the bill of the discard services are included. Every PS is an optional bill of services (BOS), and can be selected according the dominated rule. Suppose $f^{(u)}=\left[c^{(u)}, e^{(u)}\right]$ and $f^{(v)}=\left[c^{(v)}, e^{(v)}\right]$ are fitness vector solution $X^{(u)}$ and of solution $X^{(v)}$ respectively. If the condition $c^{(u)} \leq c^{(v)} \wedge e^{(u)}<e^{(v)}$ or $c^{(u)}<c^{(v)} \wedge e^{(u)} \leq e^{(v)}$ is true, vector $f^{(u)}$ dominates vector $f^{(v)}$, denoted by $f^{(u)} \prec f^{(v)}$. If solution $X^{(v)}$ is a member of the PS set, it will be replaced by solution $X^{(u)}$ correspondingly. If no dominated relationship can be found between vectors $f^{(u)}$ and $f^{(v)}$, solution $X^{(u)}$ will be added into the PS set.

\section{Part repair service configuration method}

When the overhaul service configuration is done, some parts are going to be repaired. In fact, part repair service requirements are unknown in advance, because they result from overhaul service configuration dynamically. Each part repair service can be decomposed into some part repair procedure services. Some service providers with different ability are candidates for these part repair procedure services. Outsourcing these part repair procedure 
services is a key issue. It is a multi-objective combinational optimization problem with the following considerations.

- Time consideration. Every part repair procedure service should be finished just before its due time. Both earliness and tardiness should be avoided furthest.

- Cost consideration. Every repair procedure service can be outsourced to some optional service providers with different costs. The total cost should be as less as it can be.

- Energy consumption consideration. Every repair procedure service may consume different energy by different service provider. The total energy consumption should be as less as it can be.

However, it is very difficult to balance service time, cost, and energy consumption considerations synthetically. A group of non-inferior solutions, or Pareto optimal solutions, can be found other than just a trade-off between these factors with some predefined weights. A complete BOS can be obtained by combining each the bill of repair services with the bill of exchange services and the bill of discard services. The optimization model and algorithm are two significant issues to be discussed in detail.

\subsection{The multi-objective optimization model}

Suppose $n$ part repair services are going to be configured. As to part repair service $i$, $P N_{\mathrm{i}}$ procedure services should be finished in sequence just before its due time $d t_{\mathrm{i}}$, which is an element of due time set $D T=\left\{d t_{1}, d t_{2}, . ., d t_{n}\right\}$.

Assuming $m$ service providers (SPs) are candidates, denoted by $S P=\left\{s p_{1}, s p_{2}, \ldots, s p_{m}\right\}$. As to service provider $s p_{r}$, its cost per unit time is $c_{r}$, which is an element of the unit cost set $C=\left\{c_{1}, c_{2}, \ldots, c_{m}\right\}$. The energy consumption of service provider $s p_{r}$ is $e_{r}$ per unit time, which is an element of the unit energy consumption set $E=\left\{e_{1}, e_{2}, \ldots, e_{m}\right\}$

The vector $(i, j, r)$ stands for that procedure service $j$ of part repair service $i$, defined as $p_{j}^{i}$, is going to be outsourced to service provider $s p_{r}$. A procedure service's finishing time $T E(i, j, r)$ equals to the sum of its start time $T S(i, j, r)$ and repair service time $T R(i, j, r)$.

$$
T S(i, j, r)+T R(i, j, r)=T E(i, j, r)
$$

The procedure service cost, finishing time deviation and energy consumption are subobjectives.

(1) Procedure service cost

Each service provider differs from others with its cost per unit time. A solution is regarded as a combination of service providers. The total service cost $f_{1}$ equals to the accumulation of each procedure's service cost.

$$
f_{1}=\sum_{i=1}^{n} \sum_{j=1}^{P N_{i}} T R(i, j, r) \cdot c_{r}
$$

(2) Finishing time deviation 
Based on the just-in-time (JIT) thinking, earliness and tardiness should be eliminated furthest. Then the difference between the due time and real finishing time should be minimized. The sum of squared difference, denoted as $f_{2}$, is regarded as the $2^{\text {nd }}$ subobjective.

$$
f_{2}=\sum_{i=1}^{n}\left(d t_{i}-T E\left(i, P N_{i}, r\right)\right)^{2}
$$

\section{(3) Energy consumption}

Each service provider also differs from others with its energy consumption per unit time. As to a solution, the total energy consumption $f_{3}$ equals to the accumulation of each procedure's energy consumption.

$$
f_{3}=\sum_{i=1}^{n} \sum_{j=1}^{P N_{i}} T R(i, j, r) \cdot e_{r}
$$

To achieve the multi-objective optimization goal, the fitness is a vector that equals to the minimum of these sub-objectives as fit $=\min \left[f_{1}, f_{2}, f_{3}\right]^{T}$.

In addition, the following constraints must be satisfied.

(1) Sequence Constraint: two procedure services of the same part repair service should not be operated synchronously, but orderly.

$$
\begin{aligned}
& T S\left(i, j+1, r_{1}\right)-T S\left(i, j, r_{2}\right)>\operatorname{TR}\left(i, j, r_{2}\right) \\
& i \in[1, n], j \in\left[1, P N_{i}\right], r_{1}, r_{2} \in[1, m], s p_{r_{1}}, s p_{r_{2}} \in S P
\end{aligned}
$$

(2) Resource Constraint: two procedure services cannot be done by the same service provider at the same time. A service provider that can execute two repair procedures at the same time is decomposed into two service providers.

$$
\begin{aligned}
& T S(u, v, r)-T S(i, j, r)>T R(i, j, r) \\
& i, u \in[1, n], j, v \in\left[1, P N_{i}\right], r \in[1, m], s p_{r} \in S P
\end{aligned}
$$

\subsection{The multi-objective optimization algorithm}

To find the Pareto optimum solutions, an enhanced multi-objective particle swarm optimization (MOPSO) algorithm is designed. For convenience, notations used throughout this algorithm are listed in Table 1.

Table 1 List of notations

\begin{tabular}{|l|l|}
\hline Notations & Descriptions \\
\hline $\mathrm{X}$ & Current particles' position \\
\hline Xnew & Particles' new position in current generation \\
\hline Xold & Particle's position in previous generation \\
\hline $\mathrm{M}$ & The particle number \\
\hline
\end{tabular}




\begin{tabular}{|l|l|}
\hline Pbest & The best position of each particle \\
\hline Alpha & A particle's threshold of studying to its previous position \\
\hline Beta & $\begin{array}{l}\text { A particle's threshold of studying to its historical best } \\
\text { position }\end{array}$ \\
\hline Gama & A particle's threshold of studying to the global best position \\
\hline Rbest & The best position of each iteration \\
\hline i,NC & Counters \\
\hline NC_max & The maximum iteration \\
\hline Delta & Non-dominated Solution selection probability \\
\hline
\end{tabular}

The procedure is shown in Fig. 4. Firstly, the starting position of all particles is generated randomly. The iteration continues while the counter NC is less than NC_max. In each iteration, every particle studies to its latest position, the best position in history and the global best position according to possibility. It just copies the corresponding dimension when the random decimal is smaller than the corresponding threshold, such as Alpha, Beta and Gama listed in Table 1. After a particle's fitness is calculated, it will update its best position, the global best position, and the non-dominated solutions (NDSs) in each iteration according to the domination relationship. If an iteration ends, the NDSs will be used to update the Pareto candidates (PCS) according to the domination relationship. Every particle's current position and previous position are also updated. The procedure ends when the stopping criterion is satisfied. The final PCs are obtained, while each of them is a bill of repair services. It contributes to a PS (or a BOS) when the bill of the exchange services and the bill of the discard services are included.

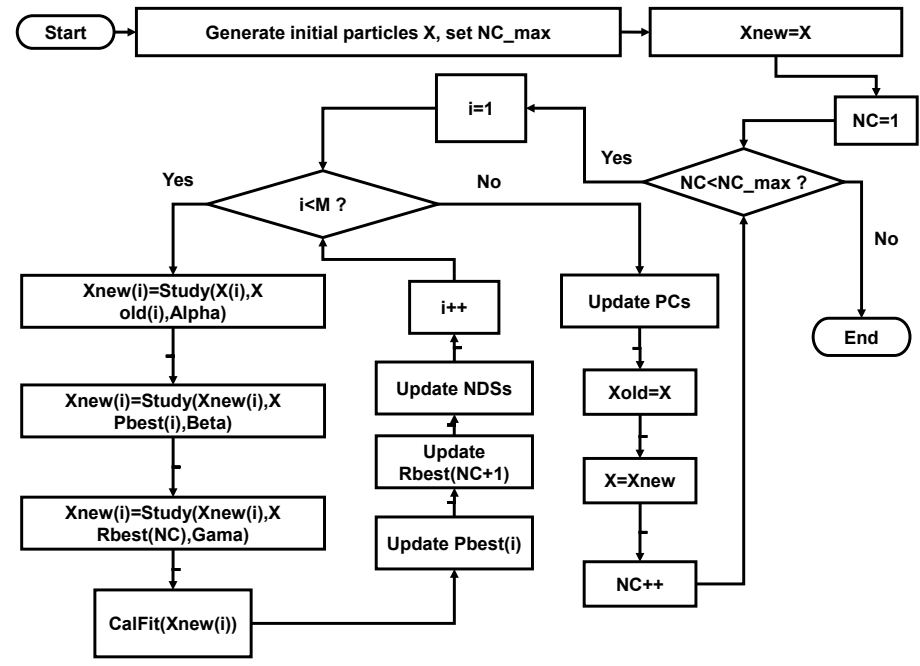

Fig. 4 Procedure of the multi-objective particle swarm optimization

Detailed discussion about the procedure is listed as follows.

1) Coding

Here, an integer coding method is used. A solution is regarded as a set $X=\left\{x_{1}, x_{2}, \cdots, x_{q}\right\}$, while $q$ is the amount of procedure service as $q=\sum_{i=1}^{n} P N_{i}$ 
The member $x_{i}$ is an element in a solution for a procedure service, and it can be defined as the following 5 -tuple array.

$$
x_{i}=\left\{s p_{r}, i, j, T S(i, j, r), T E(i, j, r)\right\}
$$

2) Initial solution generation

The pseudo-code of the initial solution generation process is shown in Fig. 5. Here, the variable Service_Provider_Time( $r$ ) is used to record the earliest available time of the service provider $s p_{r}$, and its initial value is infinite. And the variable Part_Service_Time(i) is used to record the earliest available time of part repair service $i$, and it equals to the part repair service's due time initially. All procedure services will be ordered reversely. As to procedure service $j$ of part repair service $i$, service provider $r$ is chosen randomly. The procedure service's finishing time equals to the minimum of Service_Provider_Time( $r)$ and Part_Service_Time(i). Its start time is calculated according to Eq. (8).

Every procedure service will be determined in the same way until all procedure services are settled down. Obviously, both sequence constraint and resource constraint are satisfied.

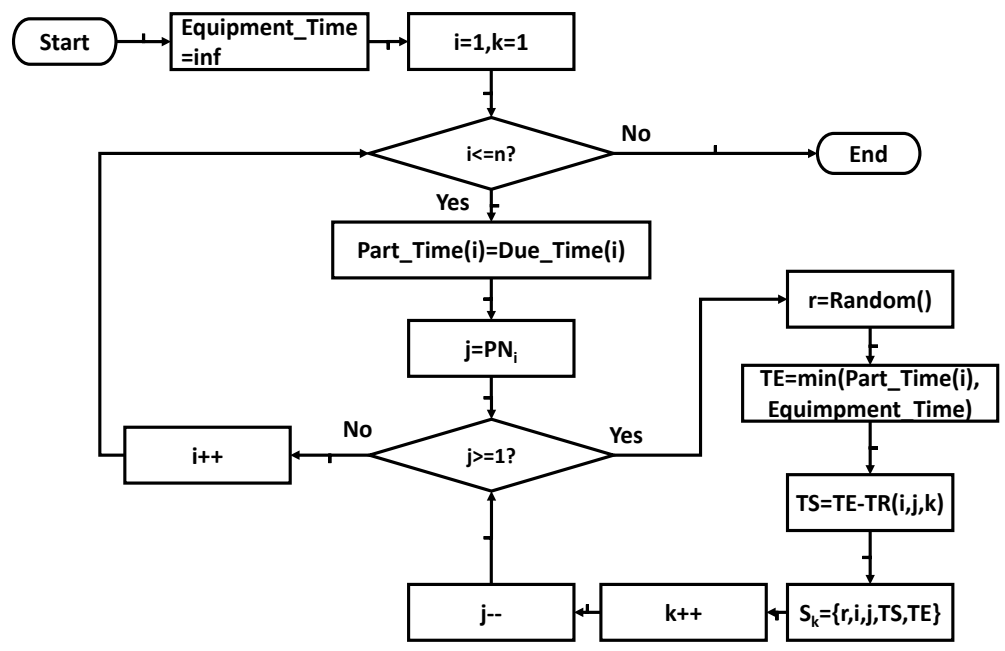

Fig. 5 The initial solution generation process

3) Domination relationship

Suppose $f^{(u)}=\left[f_{1}^{(u)}, f_{2}^{(u)}, f_{3}^{(u)}\right]^{T}$ and $f^{(v)}=\left[f_{1}^{(v)}, f_{2}^{(v)}, f_{3}^{(v)}\right]^{T}$ are fitness vectors of solution $X^{(u)}$ and $X^{(v)}$ respectively. The domination relationship between them is defined as $\forall i=[1,2,3], f_{i}^{(u)} \leq f_{i}^{(v)} \wedge \exists j=[1,2,3], f_{j}^{(u)}<f_{j}^{(v)}$, denoted by $f^{(u)} \prec f^{(v)}$. It means that vector $f^{(u)}$ dominates vector $f^{(v)}$. Therefore, solution $X^{(u)}$ replaces solution $X^{(v)}$ if $X^{(v)}$ is the best position in history or the global best position.

4) The NDS set selection rule

The NDS set is used to save non-inferior solutions generated in each iteration. As Fig. 6 shows, the domination relationship between fitness vectors of a solution and every nondominated solution in the NDS set is decided orderly. A solution will replace a nondominated solution whose fitness vector is dominated by its. If no domination relationship can be founded, the solution will replace the non-dominated solution in the NDS set according to possibility, while their Euclidean distance lies in a certain range. This policy will improve the diversity of the NDS set. 
5) The PC set selection rule

The PC set is used to save the Pareto candidates. As Fig. 7 shows, the domination relationship between the finesses of a solution from the PC set and a solution from the NDS set is decided. The solution from the NDS set will replace the solution from the PC set, while the former's fitness vector dominates the latter's.

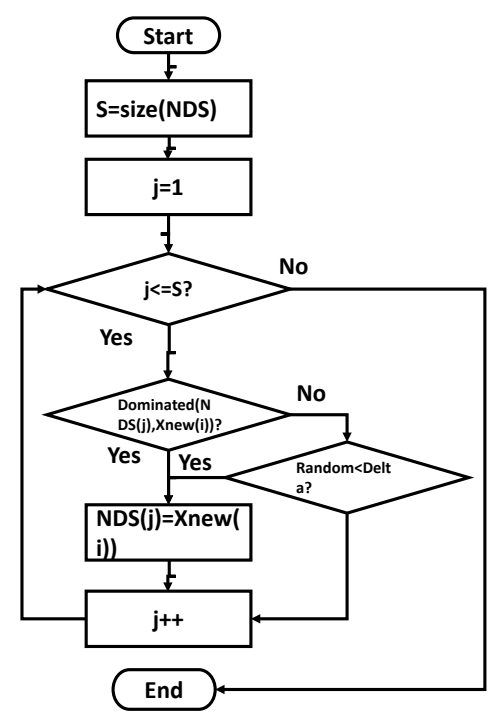

Fig. 6 The non-dominated solution set selection rule

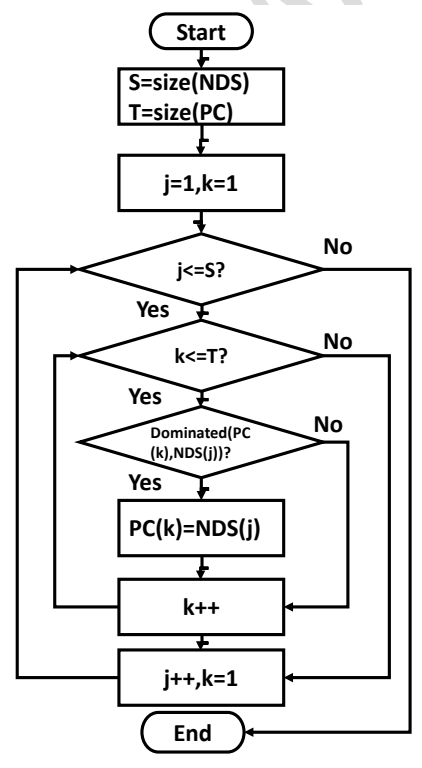

Fig. 7 The Pareto candidate set selection rule

According to the results of overhaul service configuration and part repair service configuration, some non-dominated bills of services (BOSs) can be obtained. Each of them can be used to fulfill the aero-engine overhaul service requirement with certain cost and energy consumption.

\section{Implementation of the approach as a prototype}


To verify the rigor of the proposed approach in Sections 3, 4, and 5, a prototype is implemented by extending an overhaul digitalization system (ODS). ODS was developed by the first author and his colleagues and has been used to control the aero-engine overhaul process by an aero-engine manufacturer in China for about 3 years. ODS follows the traditional product extension service mode while the overhaul service is regarded as an addon service to the aero-engine owned by the customer. Overhaul procedures supported by ODS include aero-engine disassembly, material condition check and decision-making, repair/exchange/discard execution, material recollection, aero-engine re-assembly, and testdriving. To enable sustainable use-oriented overhaul service configuration, the prototype focuses on the decision-making step based on the conditions of the engine materials. Practitioners from this aero-engine manufacturer noticed the trend of sustainable useoriented PSSs, and were interested in using and evaluating the use-oriented aero-engine overhaul service configuration approach.

To avoid any disturbances from ODS, the prototype is not integrated with ODS. Instead, the prototype runs stand-alone with a duplicated database for verification. The prototype inherits the basic bill of material (BOM) management, aero-engine disassembly, and material condition check modules from ODS. It extends ODS by supporting the use-oriented aeroengine overhaul service configuration and repair service configuration. The overhaul service configuration method is implemented by Java Server Pages (JSP). The overhaul service requirements are given via an HTML form, while the service configuration results are shown by tables in a web browser. The Java servlets read/write the database and has implemented the configuration algorithms. The repair service configuration method is implemented with Matlab $^{\circledR} 2012$ due to its data processing ability and efficient MOPSO implementation.

\section{Case study}

\subsection{Target case}

Based on the traditional add-on service mode, the overhaul service supported by ODS aimed to rebuild the aero-engine's functionality only. Every part may be repaired or discarded according to its condition. Without revealing details, the total profit is mostly determined by the number of parts discarded. Such a mode is insufficient and inefficient for the use-oriented aero-engine overhaul service.

The use-oriented aero-engine overhaul service is different. Because the aero-engines belong to the service providers, the more parts to be exchanged or repaired, the more cost and energy may be saved. Then, the service providers have incentive to decrease the number of parts discarded. To illustrate how the prototype is used for the use-oriented overhaul service, a case is chosen from overhaul services for a typical aero-engine.

\subsection{Study setting}

This case study involved the authors and practitioners. As ODS users, the practitioners have substantial knowledge and experience as well as access to needed data about the practices of overhaul services for aero-engines. The case study used the aero-engine BOM derived from ODS. In fact, the aero-engine involves nearly 10,000 parts. To verify the approach without loss of generality, a simplified BOM was used. To protect the business secrecy, the simulating data was used instead of the real data. The simulating cost and 
energy consumption were given by the practitioners according to their experiences. Regular and close collaboration between the practitioners' company and the first author's university made this data collection possible. Within the study, the prototype is used to illustrate the configuration process. The configuration results are evaluated by the practitioners.

\subsection{Results}

In this case, the flight hour requirement is 22,000 hours, and the thrust requirement is 22,700 lbf. Using the approach and prototype presented in Sections 3, 4, 5, and 6, an HSTM is built to describe the decomposition process of the aero-engine overhaul service. The overhaul service was decomposed into 47 sub-services in the component repair service layers and in the part repair service layer. Each sub-service is also formulated according to its attributions. Based on the comparison between the requirements and actual values (explained in Section 4.3), the HSTM is filtered. To save the space without losing the scientific meaning of the approach outputs, a sub-part of the filtered HSTM is shown in Fig. 8. Here, 10 decisions are to be made by the method explained in Section 4.3. As shown in Fig. 8, each decision has 2-5 service options. Then several BOSs come with the bills of exchange services, discard services and part repair services. As Fig. 8 shows, three BOSs are illustrated and compared. Their fitness vectors are $f^{(1)}=[6889481,8519.6], f^{(2)}=[6806353,8720.3]$ and $f^{(3)}=[6963329,8423.7]$ respectively. All of them are Pareto solutions because no domination relationship exists between their fitness vectors. A huge difference exists in the numbers of effective digits for the three sub-objectives, because original data of different dimensions varies greatly.

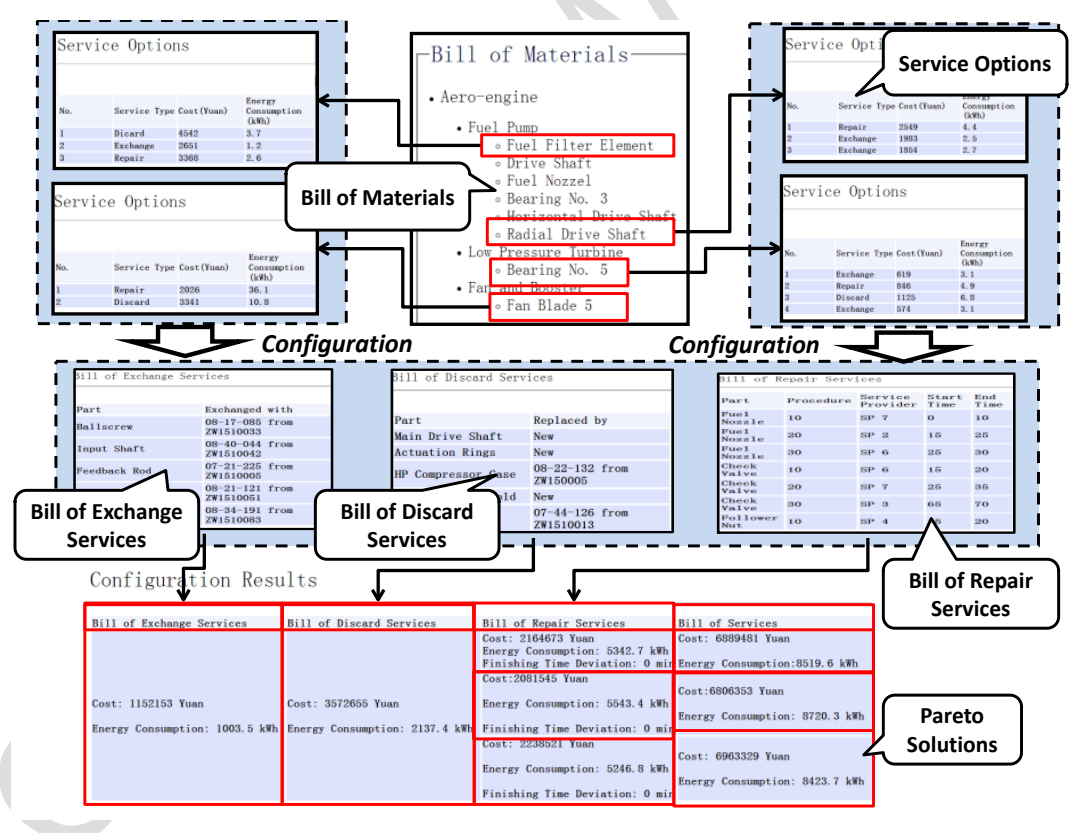

Fig. 8 The aero-engine overhaul service configuration on the case

There are 8 part repair services (PRSs) in the solution. Each part repair service has its specific due time and includes 3 or 4 repair procedure services to be outsourced. Here, 8 SPs are candidates for the par repair services. The part repair procedure service configuration method discussed above is used to find out the PC set. Some parameters are set as Alpha $=0.5$, Beta $=0.6$, Gama $=0.1, M=10$, and NC_max $=100$. After 100 iterations, some nondominated solutions are obtained. The PC set is listed in Table 2 and shown in Fig. 9(a). The service distribution of PC No. 1 is illustrated by a Gantt in Fig. 9(b). 
In Table 2, PC No. 7 costs the least, but it has the most energy consumption and the maximum finishing time deviation. On the contrary, PC No. 10 consumes the least energy, but it has relatively higher cost and finishing time deviation. They can be selected according to specific requirements. However, if the energy consumption is ignored, PC No. 3, 10 and 12 are not derived due to the others' less costs and finishing time deviation. All PCs in Table 2 are not necessarily feasible in the traditional add-on service mode, because the useoriented aero-engine overhaul service increases benefits by reducing cost and energy consumption.

Table 2 Pareto candidates of part repair services

\begin{tabular}{|c|c|c|c|}
\hline PC No. & Cost (f1, Yuan) & $\begin{array}{c}\text { Energy Consumption } \\
(\mathrm{f} 2, \mathrm{kWh})\end{array}$ & $\begin{array}{c}\text { Finishing Time } \\
\text { Deviation (f3, Min) }\end{array}$ \\
\hline 1 & 2164673 & 5342.7 & $\mathbf{0}$ \\
\hline 2 & 2081545 & 5543.4 & $\mathbf{0}$ \\
\hline 3 & 2238521 & 5246.8 & $\mathbf{0}$ \\
\hline 4 & 2095446 & 5501.5 & 5 \\
\hline 5 & 2091012 & 5489.2 & 3 \\
\hline 6 & 2231465 & 5388.7 & $\mathbf{0}$ \\
\hline$\underline{7}$ & $\mathbf{2 0 3 3 4 5 4}$ & 5501.6 & 10 \\
\hline 8 & 2154682 & 5406.4 & 8 \\
\hline 9 & 2170325 & 5242.6 & 3 \\
\hline$\underline{10}$ & 2248562 & 5156.3 & $\mathbf{0}$ \\
\hline 11 & 2101342 & 5449.1 & $\mathbf{0}$ \\
\hline 12 & 2165232 & 5312.1 & 4 \\
\hline 13 & 2186534 & 5485.5 & \\
\hline
\end{tabular}

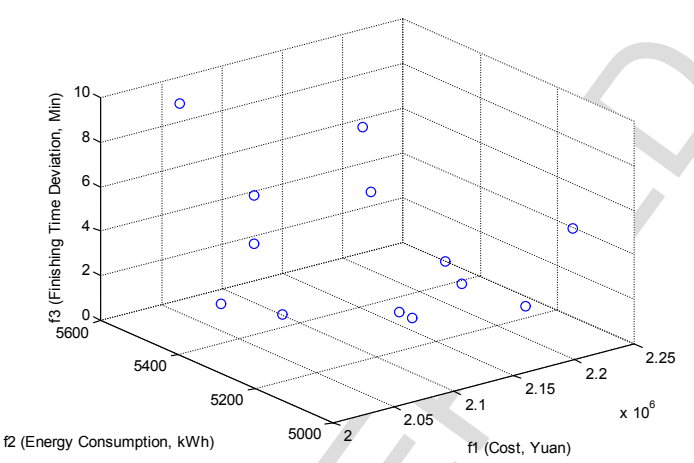

(a) The Pareto candidates' distribution

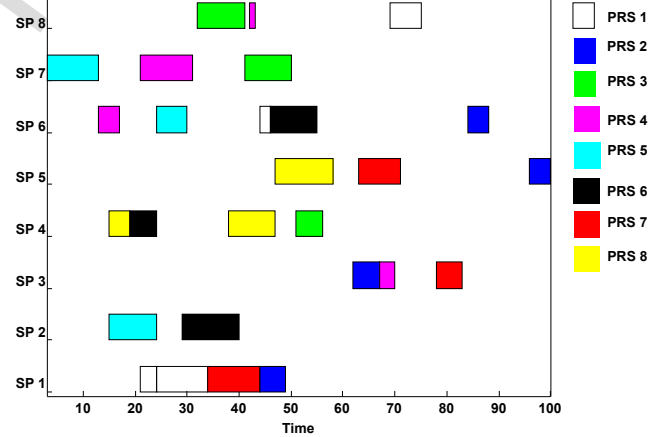

(b) The service distribution of PC No. 1

Fig. 9 Part repair procedure configuration result

\subsection{Feedback from the practitioners}

As a traditional aero-engine manufacturer, to become a sustainable PSS provider is an agenda of high interest under internal discussion. In the traditional aero-engine overhaul service supported by ODS, the engines belong to the customers. As far as the company concerns, it is safer to replace a used part with a new one. In addition, the replacement increases the company's profit. Therefore, the remaining useful life (RUL) of a part is not used at the maximum, leading to inefficiency of resource consumption.

In use-oriented PSS, the engines belong to the company. To save the cost, the company is incentivised to repair or reuse more parts. This leads to higher resource 
efficiency. However, how to increase the service life yet maintain thrust requirements is a huge challenge. The proposed approach gives an alternative solution to this challenge. The prototype has basic functions and shows potential to be used for the use-oriented aeroengine overhaul service configuration process from the practical viewpoint.

According to the configuration results, the overhaul service cost is saved while the environmental benefit is realized by reusing more parts. It is interesting particularly that the approach considers energy consumption in the configuration, because it is not the case with the traditional add-on service mode implemented in ODS. Yet, the critical fact is that the company needs to ensure the service life and thrust requirements at its own risk. To do so efficiently, RUL and conditions of every part need to be known and maintained precisely. The feasibility of the use-oriented overhaul services relies on how these data can be managed.

Although the use-oriented overhaul service is believed to be a trend in the future, its adoption in the company in a short term faces some barriers as well. The business philosophy to be associated with the use-oriented service differs from the tradition. Prior to the adoption, the business model also must be changed. In addition to the approach, a database should be built to collect and manage the conditions of reusable parts efficiently. The enterprise resource planning (ERP) system should also be redeveloped and integrated to support use-oriented aero-engine overhaul services. Thus, it is difficult to use the approach with existing aero-engines and systems. However, its adoption in new aero-engines has high potential.

\section{Discussion and conclusions}

\subsection{Discussion}

\subsubsection{Effectiveness of the proposed approach}

With the background of sustainable PSS, a use-oriented aero-engine overhaul service configuration approach is proposed in this paper. Two methods, i.e. overhaul service configuration method and part repair service configuration method, are discussed in detail. The approach has been shown effective to configure the use-oriented aero-engine overhaul service as follows.

- The approach enables to configure a use-oriented aero-engine overhaul service in a standardised manner with service requirements as input. Hierarchical trees with various sub-services to fulfil certain flight hours and thrust requirements are adopted to represent intermediate results. Every configuration result is represented as a set of BOSs. These representations make it easier for a user to understand and follow the results.

- The approach realizes to optimize the configuration considering multiple relevant objectives, i.e. the overhaul cost, the energy consumption, and the finishing time deviation. Particularly, the energy consumption is addressed as a main objective embedded in the configuration approach, and thus the environmental impact is incorporated.

8.1.2 Accuracy and robustness of the results 
All the results presented in Section 7.3 are non-dominated Pareto solutions. Namely, overhaul service cost and energy consumption are balanced furthest. They cannot be minimized simultaneously further, because there is a trade-off relation between them. Ideally, the accuracy of the results depends on their approximation to the unknown Pareto front. However, the accuracy can be verified by the fact that sub-objectives of every result are optimized and balanced as much as possible.

Due to the characteristic of multi-objective optimization, several solutions are obtained instead of a unique optimum. These solutions are resulted from the standardised procedure. Controlled by the selection rules, the non-dominated relationships among their fitness vectors always exist. This phenomenon is, however, normal in multi-objective optimization and robust against possible variance of service requirements, initial solutions, users and products.

According to the practitioners, the obtained results have sufficient accuracy and variety for practice. They also believe that every result is a feasible guideline for decomposing an aero-engine overhaul service and outsourcing related part repair services.

\subsubsection{Adopting this approach in practice in a short term}

As discussed in Section 7.4, to adopt this approach in practice, adjustments from both business and technology aspects are needed. To fit with the use-oriented PSS mode, traditional aero-engine manufacturers should reorganize their business processes. A database is also required to manage usable parts' conditions in advance. Once these conditions are met, the approach may bring its full benefits to real life. It has great potential to be adopted with new aero-engines in new business processes in a longer term according to several consulted practitioners.

Although the approach was verified by the aero-engine overhaul service, the approach holds a generic character as well. For instance, the overhaul service is also a common issue of other complex products, such as turbines, tank engines, etc. Therefore, its wider application to different industrial sectors after adjustment is expected. It should be noted that overhaul service for aero-engines is of a high level of complexity, which means the approach is capable to handle the complexity and more likely to be applicable to less complex service in principle. In addition, the multi-objective optimization is relevant especially considering the environmental requirements or the requirements from various lifecycle phases, as it makes more likely for conflicts to occur. Further, use-oriented PSSs can be motivated also by the provider to encapsulate the product from its users (Sakao et al., 2013). Therefore, the proposed approach has a high potential to be applied to a broad range of PSSs.

\subsubsection{Limitations}

The approach has limitations regarding the evaluation indexes and optimization goals in terms of environmental aspect and service network properties. These may be refined further, but consequently, the configuration model may become more complex, making it a challenge to achieve a trade-off between an increased number of objectives. A simplified BOM with 47 sub-services was used to verify the approach. In fact, an actual aero-engine includes more than 10,000 parts. The applicable scale needs to be increased to improve the practicability of the proposed approach. 
The environmental aspects addressed in the approach focused on the energy consumption. There are other environmental aspects of interest to a provider. Such an instance is resource scarcity, which is influenced by a choice of discard in a service and could be addressed in an enhanced approach based on the proposed one. In addition, developing the proposed approach further to be able to address a comprehensive set of environmental aspects including waste generation, raw material consumption, carbon emission, and water consumption is also a future work interested from the environmental sustainability viewpoint.

By using advanced smart sensing devices, many aero-engines' on-wing data can be collected. Based on these data, better cleaner production decisions can be made (Zhang et al., 2016b). Then, analyzing these data can benefit the overhaul service configuration results. Additional work to improve the approach's ability in dealing with on-wing big data should be explored.

\subsection{Conclusions}

This paper proposes a use-oriented aero-engine overhaul service configuration approach. The approach adopts a hierarchical service tree model to present the whole service activity in a comprehensible manner. It also employs a multi-objective optimization considering energy consumption as well as the repair service cost and finishing time deviation, and outputs Pareto solutions. Through a case study, the effectiveness and feasibility of the approach are verified.

The proposed approach contributes to the use-oriented aero-engine overhaul service configuration issue. It narrows the gap between the flight hours and thrust requirements and the overhaul service configuration results. The configuration process takes environmental impacts into account and adopts multi-objective evaluation and optimization algorithm. The approach can be a valuable reference for further development and application of the use-oriented aero-engine overhaul service configuration.

Some limitations described earlier call for further researches, for example adjusting the approach to further enhance its performance and applicability as well as refining the evaluation (including enhanced evaluation of the environmental aspect) as discussed in Section 8.1.4.

\section{Acknowledgements}

The research is under the support of the Natural Science Basic Research Plan in Shaanxi Province of China (Program No. 2013JM7001) and the "National Natural Science Foundation of China" (NSFC, No. 50805122). This research is also supported in part by the Mistra REES (Resource Efficient and Effective Solutions) program funded by Mistra (The Swedish Foundation for Strategic Environmental Research) and the Circularis (Circular Economy through Innovating Design) project funded by VINNOVA (Sweden's innovation agency). The authors wish to express their deep appreciation to the practitioners involved in the case study for their support. 


\section{References}

Aurich, J.C., Wolf, N., Siener, M., Schweitzer, E., 2009. Configuration of product-service systems. Journal of Manufacturing Technology Management, 20(5), 591-605

Byers, S., Groth, J., Sakao, T., 2015. Using Portfolio Theory to Improve Resource Efficiency of Invested Capital. Journal of Cleaner Production, 98, 156-165.

Cao, W., Jiang, P.Y., 2013. Modelling on service capability maturity and resource configuration for public warehouse product service systems. International Journal of Production Research, 51(6), 1898-1921.

Cherubini, S., lasevoli, G., Michelini, L., 2015. Product-service systems in the electric car industry: critical success factors in marketing. Journal of Cleaner Production 97 40-49

Chou, C.J., Chen, C.W., Conley, C., 2015. An approach to assessing sustainable productservice systems. Journal of Cleaner Production, 86, 277-284

Gaiardelli, P., Resta, B., Martinez, V., Pinto, R., Albores, P., 2014. A classification model for Product-Service offerings. Journal of Cleaner Production, 66, 507-519.

Hu, S. J., Ko, J., Weyand, L., ElMaraghy, H. A., Lien, T. K., Koren, Y., Bley, H., Chryssolouris, G., Nasr, N., Shpitalni, M., 2011. Assembly system design and operations for product variety. CIRP Ann.-Manuf.Techn. 60(2), 715-733.

Huang, B.Q., Li, C.H., Tao, F., 2014. A chaos control optimal algorithm for QoS-based service composition selection in cloud manufacturing system, Enterprise Information Systems. $8(4), 445-463$.

Huang, G.Q., Qu, T., 2008. Extending analytical target cascading for optimal configuration of supply chains with alternative autonomous suppliers. Int. J. Production Economics. 115, 39-54.

Huang, S.X., Zeng, S., Fan, Y.S., Huang, G.Q., 2011. Optimal service selection and composition for service-oriented manufacturing network. International Journal of Computer Integrated Manufacturing. 24(5), 416-430.

Liu, Y. 2013. Sustainable competitive advantage in turbulent business environments, International Journal of Production Research, 51(10), 2821-2841.

Liu, Y., Liang, L., 2015. Evaluating and developing resource-based operations strategy for competitive advantage: an exploratory study of Finnish high-tech manufacturing industries. Int. J. Prod. Res. 53(4), 1019-1037.

Long, H.J., Wang, L.Y., Shen, J., Wu, M.X., Jiang, Z.B., 2013. Product service system configuration based on support vector machine considering customer perception. International Journal of Production Research, 51(18), 5450-5468.

Joore, P., Brezet, H., 2015. A Multilevel Design Model: the mutual relationship between product-service system development and societal change processes, Journal of Cleaner Production, 97, 92-105

Kim, H.M., Michelena, N.F., Jiang, T., Papalambros, P.Y., 2000. Target cascading in optimal system design. In: Proceedings of DETC 2000: 26th Design Automation Conference, 1013 September 2000, Baltimore, MD, USA.

Kim, H.M., 2001. Target cascading in optimal system design. Ph.D. Thesis, University of Michigan, Ann Arbor, MI.

Koren, Y. 2010. The Global Manufacturing Revolution: Product-Process-Business Integration and Reconfigurable Systems, Wiley.

Meier, H., Roy, R., Seliger, G., 2010. Industrial product-service systems - IPS2. CIRP Ann. Manuf. Technol. J. 59.

Mont, O.K., 2002. Clarifying the concept of product-service system. Journal of Cleaner Production. 10(3), 237-245.

Mont, O., Dalhammar, C., Jacobsson, N., 2006. A new business model for baby prams based on leasing and product remanufacturing. Journal of Cleaner Production. 14 (17), 15091518. 
Mont, O., Tukker, A., 2006. Product-Service Systems: reviewing achievements and refining the research agenda. Journal of Cleaner Production. 14 (17), 1451-1454.

Neely, A.D., 2007. The servitization of manufacturing: an analysis of global trends. In: Proceedings of the POMS College of Service Operations and EurOMA Conference, London: London Business School.

Qu, T., Huang, G.Q., Zhang, Y.F., Dai, Q.Y., 2009. A Generic Analytical Target Cascading Optimization System for Decentralized Supply Chain Configuration over Supply Chain Grid. International Journal of Production Economics. 127(2), 262-277.

Qu, T. Huang, G.Q., Cung, V.D., Mangione, F., 2010. Optimal Configuration of Assembly Supply Chains Using Analytical Target Cascading. International Journal of Production Research. 48(23), 6883-6907.

Sakao, T., Fargnoli, M., 2010. Customization in Ecodesign: A Demand-side Approach Bringing New Opportunities? The Journal of Industrial Ecology, 14(4), 529 - 532.

Sakao, T., Rönnbäck, A.Ö., Sandström, G.Ö., 2013. Uncovering Benefits and Risks of Integrated Product Service Offerings - Using a Case of Technology Encapsulation. Journal of Systems Science and Systems Engineering, 22(4), 421 - 439.

Sakao, T., Shimomura, Y. 2007. Service engineering: a novel engineering discipline for producers to increase value combining service and product. Journal of Cleaner Production, 15(15), 590-604.

Salazar, C., Lelah, A., Brissaud, D., 2015. Eco-designing Product Service Systems by degrading functions while maintaining user satisfaction. Journal of Cleaner Production, 87, 452462

Shen, J., Wang, L., Sun, Y., 2012. Configuration of product extension services in servitisation using an ontology-based approach. International Journal of Production Research, 50(22), 6469-6488.

Smith, D.J., 2013. Power-by-the-hour: the role of technology in reshaping business strategy at Rolls-Royce. Technology Analysis \& Strategic Management, 25(8), 987-1007.

Song, W., Sakao, T., 2017. A customization oriented framework for design of sustainable Product/Service System. Journal of Cleaner Production, 140(3), 1672-1685.

Tao, F., Zhang L., Nee, A.Y.C., 2011. A Review of the Application of Grid Technology in Manufacturing. International Journal of Production Research. 49 (13), 4119-4155.

Tao, F., Laili, Y. J., Zhang, L., Zhang, Z.H., Nee, A.Y.C., 2014. QMAEA: A quantum multi-agent evolutionary algorithm for multi-objective combinatorial optimization. Simulation: Transactions of the Society for Modeling and Simulation International. 90(2), 182-204.

Tukker, A. 2004. Eight Types of Product-Service System: Eight Ways to Sustainability? Experiences from Suspronet. Business Strategy and the Environment, 13, 246 - 260.

Tukker, A., Tischner, U., 2006. Product-services as a research field: past, present and future. Reflections from a decade of research. Journal of Cleaner Production, 14, 1552-1556.

Tukker, A., 2015. Product services for a resource-efficient and circular economy - a review. Journal of Cleaner Production, 97, 76-91.

UNEP - United Nations Environment Programme, 2002. Product-service Systems and Sustainability: Opportunities for Sustainable Solutions. INDACO Department, Politecnico di Milano, Milan.

Vezzoli, C., Ceschin, F., Diehl, J.C., Kohtala, C., 2015. New design challenges to widely implement 'Sustainable Product-Service Systems'. Journal of Cleaner Production, 97, 112

Xiang, F., Hu, Y.F., Yu, Y.R., Wu, H.C., 2014. QoS and energy consumption aware service composition and optimal-selection based on Pareto group leader algorithm in cloud manufacturing system. Central European Journal of Operations Research, 22(4), 663685.

Zhang, Y.F., Liu, S., Liu, Y., Li, R., 2016a. Smart box enabled product-service system for cloud 
logistics, International Journal of Production Research, DOI: 10.1080/00207543.2015.1134840.

Zhang, Y.F., Ren, S., Liu, Y., Si, S.B., 2016b. A big data analytics architecture for cleaner manufacturing and maintenance processes of complex products, Journal of Cleaner Production, DOI: 10.1016/j.jclepro.2016.07.123

Zhu, H.H., Gao, J., Li, D.B., Tang D.B., 2012. A Web-based Product Service System for aerospace maintenance, repair and overhaul services, Computers in Industry .63, 338348. 